\title{
High order corrections to the Renormalon
}

\author{
S. V. Faleev ${ }^{1}$, P. G. Silvestrov ${ }^{1,2}$ \\ ${ }^{1}$ Budker Institute of Nuclear Physics, 630090 Novosibirsk, Russia \\ ${ }^{2}$ The Niels Bohr Institute, Blegdamsvej 17, DK-2100 Copenhagen Ø, Denmark
}

\begin{abstract}
The high order corrections to renormalon are considered. Each new type of insertions into the renormalon chain of graphs generates the correction to the asymptotics of perturbation theory of the order of $\sim 1$. However, this series of corrections to the asymptotics is not the asymptotic one (i.e. the $m$-th correction does not grow like $m$ !). The summation of these corrections for UV renormalon may change the asymptotics by factor $N^{\delta}$. For the traditional IR renormalon the $m$-th correction diverges like $(-2)^{m}$. However, this divergency has no infrared origin and may be removed by proper redefinition of IR renormalon. On the other hand for IR renormalons in hadronic event shapes one should naturally expect these multi-loop contributions to decrease like $(-2)^{-m}$. Some problems expected upon reaching the best accuracy of perturbative QCD are also discussed.
\end{abstract}

\section{Introduction}

The running coupling constant seems to be an inevitable companion of any nontrivial renormalizable field theory. In its turn the asymptotics of perturbation theory for any quantity calculated in the theory with running coupling in general is determined by renormalon [1, 2, 3, 国. The renewed interest in this kind of asymptotic estimates have been demonstrated in last few years [5, 6, 0, 8, 9, 10, 11, [12, 13]. It results even in attempts 14 to use renormalon for calculation of experimentally measurable quantities. Finally, the recent explosion of activity in the renormalon business has been stimulated by the understanding that the former may be related with the anomalously large $(\sim$ $\left.\Lambda_{Q C D} / Q\right)$ nonperturbative corrections in hadronic shape variables [15, 16, 18, 17, 19, 20].

The accurate determination of renormalon-type asymptotics turns out to be not so simple problem. It was recognised [8, 9, 10, 12, 22] that the overall normalisation of the renormalon could not be found without taking into account of all terms of the expansion of, e.g., the Gell-Mann-Low function. However, surprisingly up to now nobody have tried to sum up this series of corrections to the renormalon.

Therefore the main aim of this paper will be to discuss the possible consequences of summation of contributions from the arbitrary high order insertions to the dressed gluon line. As we demonstrate both by diagrammatic consideration and by direct analytical calculation, each new type of insertions generates the correction to renormalon of the order of $\sim 1$. However, the $k$-th correction to the asymptotics for large $k$ is not expected to have any $k$ ! enhancement. Thus at least the series of corrections to the amplitude of 
renormalon asymptotics is not the asymptotic series. On the other hand, the summation of this series leads to the sufficient change of the high order behaviour of the usual perturbative series. We show that for the UV renormalon taking into account of the high order corrections may change the $N$-th term of the perturbation theory by the factor $N^{\delta}$ (although with $\delta$ probably unknown even for QED). The $m$-th correction to the IR renormalon diverges like $(-2)^{m}$. Due to this divergency the IR renormalon does not exist in the usual sense. We propose however the proper rearrangement of the series which allows to avoid this difficulty. The IR renormalon now will be associated with certain non-Borel-summable series but the coefficients of this series themselves are the Borel-summable series in $\alpha_{s}$. Finally, the event shape renormalons, which are the most powerful among other renormalons, may be quite stable under taking into account of the high order insertions. The corresponding $m$-th correction is expected to decrease like $(-2)^{-m}$.

In accordance with their name renormalons are connected with the running of the coupling constant. Therefore in considering of the high order corrections to renormalon one is faced with the problem of renormalization scheme independent definition of the coupling. This problem may be solved quite naturally in QED. That is why, in particular, all the diagrammatic examples of this paper will be of the QED-type. The generalization to the QCD case is usually done in some heuristic way like e.g. the so called 'naive nonabelization'. Nevertheless, the first attempts to find the renormalization scheme independent definition of the effective charge in QCD have been done recently [24]. Also we will not usually make much difference between photon and gluon, thus making the tacit assumption that the difference between theories is hidden somewhere in the coefficients $b_{0}, b_{1}, b_{2}, \ldots$ of the Gell-Mann-Low equation.

The contribution of the diagrams with exchange of one soft gluon(photon) to some "physical" quantity which accounts for the infrared(IR) renormalon has the generic form

$$
R_{I R}=\int_{k \ll Q} \alpha(k) \frac{d^{4} k}{Q^{4}}
$$

The Feynman graphs corresponding to this quantity are shown in fig. 1. Similarly the

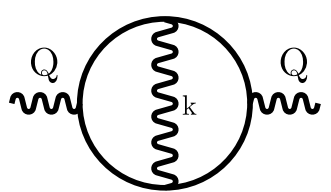

a

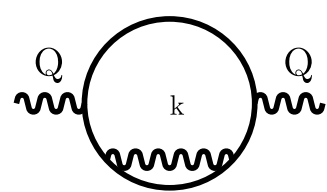

$\mathrm{b}$

Fig. 1 The renormalon graphs with exchange of one gluon. The internal gluon line will be dressed in the following figures. 
exchange of very hard gluon(photon) generates the ultraviolet(UV) renormalon

$$
R_{U V}=\int_{k \gg Q} \alpha(k) \frac{Q^{2} d^{4} k}{k^{6}} .
$$

Now only the diagram of fig. 1a contribute. During the last two years it was understood 12, 22 that the traditional UV renormalon (2) with exchange of only one hard photon does not give the largest contribution to the asymptotics. The diagrams with exchange of at least two photons turns out to be much more important. We will return to discussion of this new UV renormalon later while now our consideration of the traditional UV renormalon is of mainly methodological importance.

In (1,2) we have written down the effective running coupling constant $\alpha(k)=\alpha_{\text {eff }}(k)$, which (at least for QED, but see also [24]) is trivially connected with the transverse part of the gluon propagator. The function $\alpha(k)$ satisfies the RG equation:

$$
\frac{d \alpha}{d x}=b_{0} \alpha^{2}+b_{1} \alpha^{3}+b_{2} \alpha^{4}+\ldots, \quad x=\ln \left(Q^{2} / k^{2}\right) \quad .
$$

It is to be noted here that we have fixed the renormalization scheme by considering the effective charge. Thus our coefficients $b_{2}, b_{3}, \ldots$ are neither the free parameters, nor the known, e.g., for $\overline{M S}$ scheme, $b_{2}(\overline{M S}), b_{3}(\overline{M S})$. At first stage one may neglect $b_{1}, b_{2}, \ldots$ in (3)

$$
R_{I R}=\frac{2}{\alpha_{0}} \int_{0}^{\infty} \alpha(x) e^{-2 x} d x=\int_{0}^{\infty} \frac{e^{-2 x}}{1-b_{0} \alpha_{0} x} 2 d x=\sum_{N=0}\left(\frac{b_{0} \alpha_{0}}{2}\right)^{N} N !
$$

Here we have chosen some convenient overall normalization of the renormalon. We will consider now only the asymptotics of the perturbation theory and leave the issue of the nonperturbative ambiguity of the integral (4) due to the Landau pole to the very end of the paper. The integral (1) describes adequately the contribution of a certain chain of Feynman diagrams only for $k \ll Q$. It is seen from (4) that the main contribution to the $N$-th order of the expansion comes from $k^{2} \sim Q^{2} e^{-N / 2}$. Thus the renormalon contributions to the first few terms of perturbation theory are completely irrelevant. On the other hand, for sufficiently large $Q$ a lot of terms of the expansion (\#) come from the region $\Lambda_{Q C D}^{2} \ll k^{2} \ll Q^{2}$, where the effective charge is small and the perturbative approach for calculation of $\alpha_{\text {eff }}$ (3) seems to be useful.

Very similar calculation for the UV renormalon (2) leads to the same result as (4) up to trivial replacement $\frac{b_{0}}{2} \rightarrow-b_{0}$. This means on the one hand that the UV renormalon asymptotics in general is much more important (in $2^{N}$ times) than the IR one. On the other hand one may see that depending on the sign of $b_{0}$ the series associated with one of the two renormalons should be non-Borel summable.

\section{Diagrammatic examples}

The main part of the current interest in renormalons seems to be concentrated on the consideration of their relation with nonperturbative corrections to various observables. 


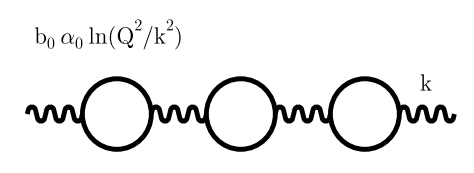

Fig. 2 The simplest chain of diagrams corresponding to the renormalization of the soft gluon line. Each bubble generates the factor $b_{0} \alpha_{0} \ln \left(Q^{2} / k^{2}\right)$.

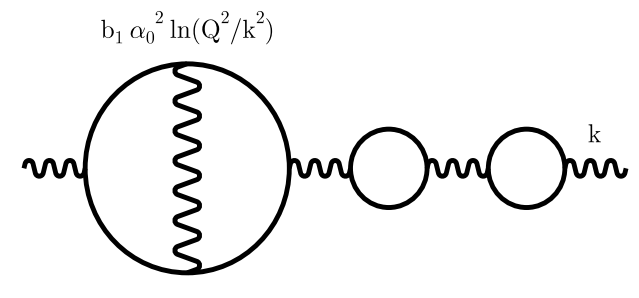

Fig. 3 The example of diagram with two - loop insertion into soft gluon line.

However, just this part of the renormalon business suffers mostly on the hard to establish assumption about the enhancement of nonperturbative corrections compared to the typical uncertainty of renormalon resummation. The only free of phenomenological input use of renormalon is for the approximate (with $1 / N$ as a small expansion parameter, where $N$ is the number of the term of the perturbative series) calculation of the high order contribution of the usual perturbation theory. Therefore, before passing to straightforward but rather formal manipulations with the RG equation (3) let us illustrate the role of complicated contributions to renormalon by the few explicit estimates of Feynman graphs.

Moreover, the experience of this diagrammatic consideration will help us in the following to distinguish, to what extent the factorial growth of the series is connected with the true infra-red(ultra-violet) physics or appears due to a simple combinatorics.

In this section we will consider only the IR renormalon. Generalization for the UV one is straightforward. The fig. 2 shows the chain of diagrams corresponding to (बi). We show only the QED-type diagrams without gluon self-interaction. Each of the $N$ bubbles from fig. 2 generates the factor $b_{0} \alpha_{0} \ln \left(Q^{2} / k^{2}\right)$ in the integrand of (11),(田). The difference between QCD and QED may be thought to be hidden in the factor $b_{0}$, accompanying the single bubble.

Now let us replace two of the simple bubbles by the more complicated diagram of fig. 3. The two loop bubbles generate the factor $b_{1} \alpha_{0}^{2} \ln \left(Q^{2} / k^{2}\right)$ in the integrand, which has one power of large logarithm less (or one $\alpha_{0}$ more) than the leading order contribution (旬). However, a large combinatorial factor $N$ appears due to a number of permutations of the second order bubble among the simple bubbles, leading to

$$
N b_{1} \alpha_{0}^{2} \ln \left(\frac{Q^{2}}{k^{2}}\right)\left[b_{0} \alpha_{0} \ln \left(\frac{Q^{2}}{k^{2}}\right)\right]^{N-2} \rightarrow\left(\frac{b_{0} \alpha_{0}}{2}\right)^{N} N ! \frac{2 b_{1}}{b_{0}^{2}} .
$$

Thus we see that taking into account one second order insertion into the soft gluon line 
leads to the correction of the order of one to the trivial asymptotics (4).

Consider now the more complicated diagram of fig. 4 with dressing of the internal gluon line of the second order bubble. To this end it is natural to write down explicitly the last integration over internal momentum of the two loop diagram

$$
b_{1} \alpha_{0}^{2} \int_{k^{2}}^{Q^{2}}\left[b_{0} \alpha_{0} \ln \left(\frac{Q^{2}}{q^{2}}\right)\right]^{n} \frac{d q^{2}}{q^{2}}=\frac{1}{n+1} b_{1} \alpha_{0}^{2} \ln \left(\frac{Q^{2}}{k^{2}}\right)\left[b_{0} \alpha_{0} \ln \left(\frac{Q^{2}}{k^{2}}\right)\right]^{n} .
$$

Thus up to the overall factor $\frac{1}{n+1}$ the contribution of diagram of fig. 4 coincides with

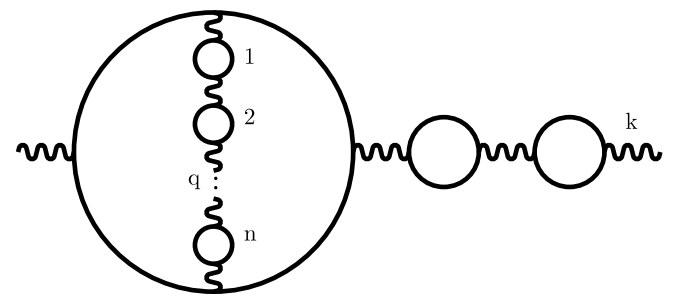

Fig. 4 The dressing of internal gluon line of the second order bubble by $n$ simple bubbles.

that of fig. 3. Summation over $n$ naturally leads to $\ln (N)$. Taking into account a number

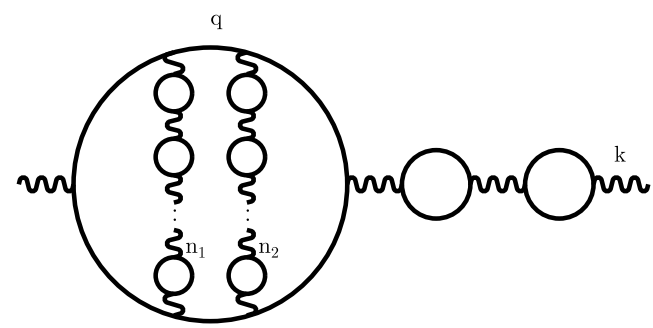

Fig. 5 Three loop insertion with dressing of two internal gluon lines by the simple chains of bubbles. The summation over $n_{1}$ and $n_{2}$ allows to compensate all extra $\alpha$-s.

of large bubbles of fig. 4 allows to exponentiate the correction

$$
\left(\frac{b_{0} \alpha_{0}}{2}\right)^{N} N ! \exp \left(\frac{2 b_{1}}{b_{0}^{2}} \ln (N)\right)=\left(\frac{b_{0} \alpha_{0}}{2}\right)^{N} N^{\frac{2 b_{1}}{b_{0}^{2}}} N !
$$

This is the generally recognised expression for the IR renormalon. Our argumentation up to this stage repeats the line of reasoning of the paper [0]. However, the argument of the exponent in (7) was found with the $\sim 1 / \ln (N)$ accuracy and therefore the nontrivial overall factor as well as the function of $N$, weaker than $N^{\gamma}$, may appear in (7).

Now let us consider the three loop correction (fig. 5 with $n_{1}=n_{2}=0$ ). This contribution generates the factor $b_{2} \alpha_{0}^{3} \ln \left(Q^{2} / k^{2}\right)$ in the integrand of (4). Thus here we 
have two extra $\alpha_{0}$ which at first glance could not be compensated by one combinatorial $N$ and hence the diagram of fig. 5 seems to generate only the $\sim 1 / N$ correction to renormalon. However, let us see, what happens if one dresses the internal gluon lines of the three loop diagram. Now summation over the number of trivial insertions $n_{1}, n_{2}$ gives:

$$
b_{2} \alpha_{0} \ln \left(\frac{Q^{2}}{k^{2}}\right) \sum_{n_{1}, n_{2}} \alpha_{0}^{2} \frac{N-n_{1}-n_{2}-2}{n_{1}+n_{2}+1} \sim b_{2} \alpha_{0} \ln \left(\frac{Q^{2}}{k^{2}}\right) \times\left(\alpha_{0} N\right)^{2} .
$$

Here the factor $\left(n_{1}+n_{2}+1\right)^{-1}$ appears after integration over the internal momentum of the large bubble, while $\left(N-n_{1}-n_{2}-2\right)$ accounts for the combinatorics. We see that after dressing of all gluon lines the three loop $\left(\sim b_{2}\right)$ diagram generates the correction to renormalon of the order of $\sim 1$. One can easily show that four loop $\left(\sim b_{3}\right)$, five loop $\left(\sim b_{4}\right)$ etc. diagrams generate the corrections of the same order of magnitude. Previously the analogous proof of the importance of the high loop corrections was done by Mueller 21] but this result was not published.

\section{Upon summation of the corrections for the UV renormalon}

It is easy to integrate formally the renormalization group equation (3)

$$
-\frac{1}{\alpha}+\frac{1}{\alpha_{0}}-\frac{b_{1}}{b_{0}} \ln \left(\frac{\alpha}{\alpha_{0}}\right)-c_{2}\left(\alpha-\alpha_{0}\right)-c_{3}\left(\alpha^{2}-\alpha_{0}^{2}\right)-\ldots=b_{0} x
$$

where, $c_{2}=b_{2} / b_{0}-b_{1}^{2} / b_{0}^{2}, c_{3}=\left(b_{3} / b_{0}-2 b_{2} b_{1} / b_{0}^{2}+b_{1}^{3} / b_{0}^{3}\right) / 2, \ldots$. For arbitrary $k$ one has $c_{k}=\left(b_{k} / b_{0}-\ldots\right) /(k-1)$. This "exact" solution still is too informative for us. First of all, most of the terms containing $\alpha_{0}$ in (9), namely $c_{2} \alpha_{0}, c_{3} \alpha_{0}^{2}, c_{4} \alpha_{0}^{3}, \ldots$ will contribute only to the $\sim 1 / N$ corrections to the asymptotics. Therefore we may write

$$
\alpha=\frac{\alpha_{0}}{1-b_{0} \alpha_{0} x-\left(b_{1} / b_{0}\right) \alpha_{0} \ln \left(\alpha / \alpha_{0}\right)-c_{2} \alpha \alpha_{0}-c_{3} \alpha^{2} \alpha_{0}-\ldots} .
$$

Let us introduce now the truncated running coupling

$$
\alpha_{t}=\frac{\alpha_{0}}{1-b_{0} \alpha_{0} x-\left(b_{1} / b_{0}\right) \alpha_{0} \ln \left(\alpha_{t} / \alpha_{0}\right)} .
$$

Now one may again formally expand the exact $\alpha(10)$ in the series

$$
\alpha=\alpha_{t}\left(1+\beta_{2} \alpha_{t}^{2}+\beta_{3} \alpha_{t}^{3}+\ldots\right)
$$

The explicit formulas for a first few coefficients $\beta_{k}$ may be easily found. However we will be interested only in the large- $k$ asymptotic behaviour of $\beta_{k}$. Naturally for our definition of the effective charge (3) the coefficients $b_{k}$ themselves form the asymptotic series $b_{k} \sim k$ !. Due to that one easily finds the asymptotics of $\beta_{k}$ in (12).

$$
\beta_{k}=\frac{b_{k}}{k b_{0}}\left(1+O\left(\frac{1}{k}\right)\right) .
$$


In the previous section we have shown diagrammatically that contributions to the asymptotics from the high order terms of the RG equation (3) $\sim b_{2}, b_{3}, b_{4}, \ldots$ (or now $\sim \beta_{2}, \beta_{3}, \beta_{4}, \ldots$ (12)) are not small. However, the contribution induced by the second (two loop) term $b_{1} \alpha^{3}$ play an outstanding role due to the additional enhancement by $\ln (N)$. This is the reason for taking the truncated coupling $\alpha_{t}$ (11) as the expansion parameter in (12).

The expression (11) itself is the transcendental equation for function $\alpha_{t}\left(\alpha_{0}\right)$ which may be solved iteratively. In ref. [23 the IR renormalon asymptotics with $\alpha_{\text {eff }}$ in (11) replaced by the only truncated $\alpha_{t}$ (11) has been considered in details. This calculation turns out to be surprisingly sophisticated. For any finite number of iterations in the transcendental equation (11) the asymptotics contains some new functional dependence on $N$ compared to the usual IR renormalon (7). The generally accepted result is restored only after performing the infinite number of iterations in (11). However, we will skip now the discussion of the role of the first few terms of the series (12) and concentrate our attention on $\beta_{k}$ with very large $k$. In this case it will be enough to make only one iteration in (11) (namely $\ln \left(\alpha_{t} / \alpha_{0}\right)=-\ln \left(1-b_{0} \alpha_{0} x\right)$ in (11)).

Consider first the UV renormalon. Moreover, let us restrict ourselves on the UV renormalon for pure QED. The first important observation (see [12, 23]) is that the series of corrections to renormalon generated by (12) although have no any reasonable small parameter but also is not the asymptotic series. In order to show that it is so consider for the moment the simplified version of (11, 12) with $b_{1}=0$. Now one has immediately for the UV renormalon (2)

$$
\sum_{m} \frac{1}{\alpha_{0}} \int_{0}^{\infty} \beta_{m}\left(\frac{\alpha_{0}}{1+b_{0} \alpha_{0} y}\right)^{m+1} e^{-y} d y=\sum_{m} \alpha^{N}\left(-b_{0}\right)^{N} N ! \frac{\beta_{m}}{\left(-b_{0}\right)^{m} m !} .
$$

We see that though $\beta_{m}$ themselves are determined by the UV renormalon and therefore have the form $\beta_{m} \sim m^{\gamma}\left(-b_{0}\right)^{m} m$ ! the two most dangerous factors from the $\beta_{m}$, the $m$ ! and $\left(-b_{0}\right)^{m}$, have been removed from the series.

Up to now we have chosen rather arbitrarily the overall normalization of renormalon. In order to go further in understanding the role of high order corrections to UV renormalon chain we have to specify the normalization. Consider following ref. [12] (and almost everybody others in the renormalon business) the correlation function of two electromagnetic currents (see also the eqs. (1,2) of ref. 112 connecting this quantity with $R_{e^{+} e^{-} \rightarrow \text { hadrons }}$ )

$$
\Pi_{\mu \nu}=i \int d x e^{i q x}\left\langle 0\left|T\left\{j_{\mu}(x) j_{\nu}(0)\right\}\right| 0\right\rangle=\left(q_{\mu} q_{\nu}-g_{\mu \nu} q^{2}\right) \Pi\left(Q^{2}\right)
$$

We will be naturally interested in $\Pi\left(Q^{2}\right)$ in the Euclidean domain.

The simple calculation of the diagram fig. 1a for $k \gg Q$ gives

$$
\Pi\left(Q^{2}\right)=\frac{N_{f}}{12 \pi^{2}}\left\{\ln \left(\frac{\mu^{2}}{Q^{2}}\right)+\ldots-\frac{1}{3 \pi} \int \alpha(k) \ln \left(\frac{k^{2}}{Q^{2}}\right) \frac{Q^{2} d k^{2}}{k^{4}}\right\} .
$$


Here the first term in brackets is the parton model prediction, while the integral is expected to generate the asymptotic series of the perturbation theory. One may substitute into (16) the one loop running coupling constant and easily reproduce the "traditional" (before [12, 22]) UV renormalon [25]. By dots in (16) we have denoted the rest part of the perturbative series which is not included into the leading UV renormalon (and is expected to be much smaller than the UV renormalon).

The polarization operator $\Pi$ is trivially connected with the $\beta$-function for the effective charge (3)

$$
4 \pi\left(Q^{2} \frac{d \Pi}{d Q^{2}}\right)_{\mu^{2}=Q^{2}}=\sum b_{n}[\alpha(Q)]^{n} .
$$

However, because we are interested only in the asymptotics we may find directly from the equation (3) that

$$
\frac{d}{d \ln \left(Q^{2}\right)} n !\left(-b_{0} \alpha\right)^{n}=(n+1) !\left(-b_{0} \alpha\right)^{n+1}\left(1+O\left(\frac{1}{n}\right)\right) .
$$

Thus for our purposes

$$
4 \pi \Pi\left(Q^{2}\right)=\sum b_{n}[\alpha(Q)]^{n}
$$

Now we have to substitute the series for the effective charge $\alpha(k)$ (12) into the integral (16) and expand the result in series in $\alpha(Q)$. The effective method which allows to find the coefficients of such expansion was developed in [23]. First of all it is convenient to introduce the new variables

$$
a=-b_{0} \alpha_{0} \quad, \quad \beta=-b_{1} / b_{0}^{2} \quad .
$$

For QED $b_{0}=-\frac{N_{f}}{3 \pi}, b_{1}=-\frac{N_{f}}{4 \pi^{2}}$ and $\beta=\frac{9}{4 N_{f}}$. With this new variables the truncated effective charge in the first nontrivial approximation takes the form

$$
\alpha_{t}=-\frac{1}{b_{0}} \frac{a}{1-a x+\beta a \ln (1-a x)} \quad ; \quad x=\ln \left(\frac{k^{2}}{Q^{2}}\right) .
$$

The contribution of the $n$-th term of the formal expansion (12) to the polarization operator $(16)$ is of the form

$$
\Pi_{n}=\frac{-\beta_{n}}{12 \pi^{2}\left(-b_{0}\right)^{n}} \int_{0}^{\infty}\left[\frac{a}{1-a x+\beta a \ln (1-a x)}\right]^{n+1} x e^{-x} d x .
$$

Note that this is the $n$-th term of the expansion in the series in truncated running coupling $\alpha_{t}(k)$ and it still contains the whole series in $\alpha(Q)$. As before we are looking for the $N$-th term of the series in $\alpha(Q)$. Below we will see that the most important contribution comes from $n \sim N$. The simple binomial expansion now gives

$$
\begin{aligned}
\Pi_{n}= & \frac{-\beta_{n}}{12 \pi^{2}\left(-b_{0}\right)^{n}} \int\left[\frac{a}{1-a x}\right]^{n+1} \sum_{m=0} \frac{(n+m) !}{m ! n !}\left[\frac{-\beta a \ln (1-a x)}{1-a x}\right]^{m} x e^{-x} d x= \\
= & \frac{-\beta_{n}}{12 \pi^{2}\left(-b_{0}\right)^{n}} \int \frac{a^{n}}{(1-a x)^{n+1}} \sum_{m=0} \frac{(n+m) !}{m ! n !}\left[\frac{-\beta a \ln (1-a x)}{1-a x}\right]^{m} e^{-x} d x .
\end{aligned}
$$


Here in the second equality we have used that

$$
\frac{a x}{1-a x}=\frac{1}{1-a x}-1
$$

and than have neglected the -1 (one may easily see that this less singular contribution will lead to the $\sim 1 / N$ correction to the asymptotics).

For estimation of the $N$-th order contribution of perturbation theory we will use the formula for $N$-th term of the expansion of the integral in powers of $a$

$$
\left\{\int \frac{e^{-t} d t}{1-a t}\left[\frac{\beta a}{1-a t}\right]^{k}\left[\ln \frac{1}{1-a t}\right]^{m}\right\}_{N}=a^{N} N ! \frac{\beta^{k}}{k !}\left[\ln \frac{N}{k}\right]^{m} .
$$

Here both $m$ and $k$ are supposed to be large. In order to derive (25) one has to use the asymptotics of gamma-function together with the trivial identity

$$
(\ln (p))^{n}=\lim _{\varepsilon \rightarrow 0}\left(\frac{\partial}{\partial \varepsilon}\right)^{n} p^{\varepsilon} .
$$

Formula (25) allows now to calculate the $N$-th term of the expansion of (23) in series in $\alpha_{0}^{N}\left(a^{N}, a=-b_{0} \alpha_{0}\right)$

$$
\begin{aligned}
\left\{\Pi_{n}\right\}_{N} & =\frac{-\beta_{n}}{12 \pi^{2}\left(-b_{0}\right)^{n}} a^{N} N ! \sum_{m=0} \frac{\beta^{m}}{(n+m) !} \frac{(n+m) !}{m ! n !}\left[\ln \frac{N}{n+m}\right]^{m}= \\
& =-\frac{1}{12 \pi^{2}}\left(-b_{0} \alpha_{0}\right)^{N} \frac{\beta_{n}}{\left(-b_{0}\right)^{n} n !}\left(\frac{N}{n}\right)^{\beta} .
\end{aligned}
$$

Here we have neglected $m$ compared to $n$ in the argument of logarithm because effectively $m \sim \ln (N)$ while as we will see in the moment $n \sim N$. Combining together $(19,27)$ and the asymptotics of $\beta_{k}$ (13) one finds the equation for $b_{N}$

$$
b_{N}=-\frac{1}{3 \pi b_{0}}\left(-b_{0}\right)^{N} N^{\beta} N ! \sum_{n<N} \frac{1}{n} \frac{b_{n}}{\left(-b_{0}\right)^{n} n^{\beta} n !} .
$$

Here $\beta=-b_{1} / b_{0}^{2}=9 /\left(4 N_{f}\right)$ for QED. This equation is even further simplified after substitution

$$
\begin{aligned}
& b_{N}=\left(-b_{0}\right)^{N} N^{\beta} N ! c_{N}, \\
& c_{N}=\sum_{n<N} \frac{c_{n}}{n\left(-3 \pi b_{0}\right)}=\frac{1}{N_{f}} \sum_{n<N} \frac{c_{n}}{n}\left(1+O\left(\frac{1}{n}\right)\right) .
\end{aligned}
$$

The solution to this last equation is evident $c_{N}=$ const $\times N^{1 / N_{f}}$ and for $b_{N}$ one has

$$
b_{N}=\operatorname{const}\left(-b_{0}\right)^{N} N^{\frac{9}{4 N_{f}}+\frac{1}{N_{f}}} N ! .
$$

So we arrived at the surprising result: taking into account all possible insertions to the renormalon chain allowed to change the power of $N$ in the asymptotics, which for 
many years was thought to be determined by only the two terms of the Gell-Mann-Low $\beta$-function $b_{0}$ and $b_{1}$.

The overall constant in (30) could not be found in closed form. In terms of the equation (29) this constant is determined by the initial condition at $N \sim 1$. But for $N \sim 1$ the equation (29) is no more valid as we have indicated explicitly by writing $(1+O(1 / n))$.

This reduction to the initial value problem shows how one may reformulate the problem of the overall normalization of renormalon. Finally, the solution looks almost like tautology. One is allowed to look for the renormalon asymptotics in the form

$$
R_{N}=A_{n} N^{\delta}\left(-b_{0}\right)^{N} N !
$$

Here $n$ is the number of terms of the perturbation theory which were calculated explicitly $(n<N)$. The normalization constant $A_{n}$ may be found with only the $\sim 1 / n$ accuracy. This is the important difference between the renormalon and instanton [26] induced asymptotics. For instantons not only the overall amplitude of the asymptotics is known

but also the $\sim 1 / N$ corrections to this asymptotics were considered [28, 29] (see also [27]).

However, one may consider the renormalon calculus only as a way to extend the explicit perturbative calculations by one more approximate term (and this is the most straightforward application of renormalon). In this approach the equation (31) still is quite informative. It shows that after the explicit calculation of $N$ terms of perturbation theory one will immediately found the $N+1$-st term with at least the $\sim 1 / N^{2}$ accuracy.

\section{UV renormalons with many hard photons}

Our formula (30) for asymptotics of the coefficients $b_{N}$ would be a nice new result if published 3-years ago. However, as we have told in the introduction Vainshtein and Zakharov in their preprint of April 1994 [12 have shown that the traditional UV renormalon (2) (fig. 1a) does not give the largest contribution to the asymptotics. They have found a series of new diagrams (starting from two three-loop diagrams) which generate the asymptotics much larger than (2). The authors of [12] have used the OPE in order to find the contribution to polarization operator of these new diagrams (see Parisi [2], who first proposed to use the OPE for renormalon calculus). Finally, the new result for $\Pi\left(Q^{2}\right)$ reads

$$
\Pi_{U V}\left(Q^{2}\right)=\text { const } \int_{k>Q}\left(\frac{\alpha(k)}{\alpha(Q)}\right)^{2+\gamma} \frac{Q^{2} d^{4} k}{k^{6}}
$$

with $\gamma$ for the QED case

$$
\gamma=\frac{3}{N_{f}}\left(\sqrt{\left(\frac{2 N_{f}+1}{6}\right)^{2}+\frac{11}{4}}-\frac{2 N_{f}+1}{6}\right) .
$$


Now it is easy to substitute the two loop running coupling (11,21) into (32) and find the UV renormalon of the form (31) with

$$
\delta=1+\gamma+\frac{9}{4 N_{f}}
$$

However, it is clear that this result for $\delta$ will be changed immediately if one substitutes the all-loop $\alpha_{\text {eff }}$ into (32) and repeats the simple calculation which we have performed in the previous section for $\Pi\left(Q^{2}\right)(16)$. The magnitude of the correction to $\delta$ (34) now will depend on the explicit value of the overall normalization of (32) which may be extracted from [12, 22].

On the other hand, if we are going to substitute the exact running coupling into (32) we should also take into account all possible corrections to this formula

$$
\Pi_{U V}\left(Q^{2}\right)=\text { const } \int_{k>Q}\left(\frac{\alpha_{e f f}(k)}{\alpha(Q)}\right)^{2+\gamma}\left(1+\sum \pi_{m} \alpha_{e f f}^{m}(k)\right) \frac{Q^{2} d^{4} k}{k^{6}}
$$

If the coefficients here behave like

$$
\pi_{m} \sim \frac{b_{m}}{m}
$$

for large $m$, taking into account of this series will change again the exponent $\delta$ (34, 31). Moreover, if $\pi_{m}$ grows faster with $m$ than (36) it will be a catastrophe for asymptotics as may be seen from (29). The eq. (36) is likely the upper bound for the coefficients of the expansion (35).

Unfortunately we do not see now the clear way to estimate the asymptotics of the coefficients $\pi_{m}$ in (35). It is rather probable that the corrections to $\delta$ due to the $\pi_{m}$ and $\alpha_{\text {eff }}(k)^{2+\gamma}$ will compensate each other and the result of refs. [12, 22] will be restored. Furthermore, the authors of [12] have demonstrated in their conclusions that they are ready to meet any surprise from the high order corrections to the renormalon. Therefore, even if such compensation do not take place and $\delta$ (34) is changed this result will not be in complete disagreement with [12].

Anyway, after one finds the new $\delta$ from (35) the overall normalization of the UV renormalon will be found with only $\sim 1 / n$ accuracy and only after the explicit calculation of the first $n$ terms of the series. We write here $\sim 1 / n$ although it may be some power of it $\sim(1 / n)^{k}$. Moreover, may be just the better understanding of the high order contributions to renormalon like in (35) may help to estimate, with what accuracy one may found the $n+1$-st term of perturbation theory after explicit calculation of $n$ terms?

\section{$5 \quad$ High order corrections for the IR renormalon and the best accuracy of perturbative QCD}

The IR renormalon have attracted much more interest during the last few years than the UV one. This conclusion may be drawn even by simple counting of the number of 
publications. It seems that most of the authors do not consider the UV renormalon as renormalon at all.

The reason for such asymmetry is quite evident. In QCD the UV renormalon is Borel summable while the IR renormalon is not. Physically this non-Borel-summability means that depending on the details of chosen resummation procedure the obtained predictions for observables will vary by some power corrections. Namely for the case of light fermions and inclusive cross sections this correction will be of the form $\sim\left(\Lambda_{Q C D} / Q\right)^{4}$. There is absolutely no way to find the amplitude of this nonperturbative $\sim Q^{-4}$ contribution from the perturbative renormalon calculus.

If there is no rigourous way to calculate the $\sim\left(\Lambda_{Q C D} / Q\right)^{4}$ correction one may try at least to extract them from the comparison of theoretical prediction with the experiment. Naively one may take say the experimental value of $R_{e^{+} e^{-} \rightarrow \text { hadrons }}$ subtract the partonmodel contribution and $2-3$ known $\sim \alpha_{s}$ corrections and look for the power corrections. However, this procedure certainly will not work at least for sufficiently large $Q$ 't. The more or less reasonable procedure is the following: one has to calculate a huge number

$$
N_{I R}(Q)=\frac{2}{b_{0} \alpha(Q)}
$$

terms of perturbation theory $\left(N_{I R}\right.$ is the function of $Q$ !) and subtract them from the experimental ratio $R(Q)$. The rest will be the needed $(\Lambda / Q)^{4}$ correction.

The first problem (if not worry about the terrible analytical calculations!) which will encounter us upon performing this procedure is the UV renormalon. The series for UV renormalon blows up at $N=1 /\left(b_{0} \alpha(Q)\right)$ - twice before the critical value (37). However the way to avoid this problem seems to be rather clear. As we have seen (1,2) the UV and IR renormalons originate from the very different regions of variation of the internal momentum in the diagram ( $k$ in the fig. 1 ). Naturally one may divide the integral into two parts $k<Q$ and $k>Q$ and than obtain the result in the form

$$
\Pi=\sum_{U V}\left(-b_{0} \alpha\right)^{N} N !+\sum_{I R, N<N_{I R}}\left(\frac{b_{0}}{2} \alpha\right)^{N} N !
$$

Here the first series is much larger than the second one but allows the explicit(Borel) summation. So one has to sum up exactly the series for the UV renormalon in (38) and then subtract this resummed contribution from the experimental value of $R_{e^{+} e^{-} \rightarrow \text { hadrons }}$.

Our goal is to reach the best accuracy of the perturbative QCD $\sim(\Lambda / Q)^{4}$. Therefore the summation of the UV renormalon in (38) also should be done with $\sim(\Lambda / Q)^{4}$ accuracy. This is also not so easy to do because even the smallest term of the series for the UV renormalon is of the order of $\sim(\Lambda / Q)^{2}-$ much larger than the accuracy we want. For example, if one simply substitutes the 1-loop $\alpha(k)$ into naive renormalon (16)

\footnotetext{
${ }^{1}$ the success of the QCD sum rules approach at low $(\sim 1 \mathrm{GeV})$ energies is usually considered as the indication that the actual power corrections in QCD have some additional enhancement compared to the typical uncertainty of renormalon resummation. However, this enhancement will became less and less important at high energies which only may be a subject of perturbative QCD.
} 
the accuracy of the resummed value will be only $O\left(\alpha^{2}\right)$ and so on. It is quite probable that in order to sum up the UV renormalon with the $(\Lambda / Q)^{4}$ accuracy one still has to calculate exactly about $N_{I R}=2 /\left(b_{0} \alpha\right)$ terms of the series.

However, suppose that accurate enough summation of the UV renormalon in (38) has been done. Let us see, what can we say about the IR-renormalon part of the polarization operator in view of our experience of working with high order corrections to renormalon chain? The corresponding contribution to $\Pi(15-19)$ now has the form of the integral (四). Simply repeating the calculations (22-28) one gets

$$
\left\{\Pi_{I R}\right\}_{N}=\operatorname{const}\left(\frac{b_{0}}{2} \alpha\right)^{N} N^{-1+2 b_{1} / b_{0}^{2}} N ! \sum_{n} \frac{\beta_{n}}{\left(b_{0} / 2\right)^{n} n^{2 b_{1} / b_{0}^{2} n !}} .
$$

But $\beta_{n} \sim b_{n}$ now is determined by the first UV renormalon and thus $\beta_{n} \sim\left(-b_{0}\right)^{n} n$ ! . Therefore we see that the sum of the multiloop corrections to the IR renormalon leads to the correction of the relative order of magnitude

$$
\sim \sum_{n} \frac{\beta_{n}}{\left(b_{0} / 2\right)^{n} n !} \sim \sum(-2)^{n}=\infty
$$

The series of the corrections to renormalon is not asymptotic, but is ugly divergent. It looks like some interference of the IR and UV renormalons.

Nevertheless, as we will see now the divergency itself of the series (40) is not connected with the infrared physics. As we saw in (14) the $N$ ! in the leading contribution to the IR renormalon appears simply due to the $N$-th power of the large logarithm $x^{N}=\ln \left(Q^{2} / k^{2}\right)^{N}$. Therefore as we have told after the eq. (4) this trivial renormalon contribution falls down into the infrared momentum region like $k^{2} \sim Q^{2} e^{-N / 2}$. On the other hand, as we have seen in the section 2 for high order corrections to the renormalon chain the same $N$ ! appears not only due to the power of logarithm but also due to the more and more complicated combinatorics. This change in the origin of the $N$ ! would not be taken into account if the series of corrections to renormalon was convergent. However, for divergent series of the kind of (40) one should naturally to reformulate the method of calculation of IR renormalon in order to be able to control from what distances each contribution came. To this end let us rewrite the renormalon (II) in terms of the series in the powers of $\ln \left(Q^{2} / k^{2}\right)$

$$
\begin{aligned}
\Pi_{I R}(Q) \sim \int \frac{k^{2} d k^{2}}{Q^{4}} \alpha(k) & =\sum_{n} \int \frac{k^{2} d k^{2}}{Q^{4}} \alpha(Q)^{n+1}\left[b_{0} \ln \left(\frac{Q^{2}}{k^{2}}\right)\right]^{n} F_{n}(\alpha(Q)) \\
& =\sum_{n} \alpha(Q)^{n+1} F_{n}(\alpha(Q))\left(\frac{b_{0}}{2}\right)^{n} n !
\end{aligned}
$$

Now all the high order corrections to renormalon chain are hidden in $F_{n}(\alpha)$. The function $F_{n}(\alpha)$ itself may be expanded in the asymptotic series. But it would be the Borel summable series.

In the previous two sections we have tried to show that there is a lot of open questions concerning the UV renormalon. Therefore, now we will illustrate only by the simple toy 
example how the functions $F_{n}(\alpha)$ (41) will be found after one solves all this "ultraviolet problems". Suppose the running coupling $\alpha(k)$ in (41) is described by the simplest Borel integral

$$
\begin{aligned}
& \alpha(k)=\int_{0}^{\infty} \frac{\tilde{\alpha}}{1+b_{0} \tilde{\alpha}(x) y} e^{-y} d y=\tilde{\alpha} \sum_{n=0}^{\infty}\left(-b_{0} \tilde{\alpha}\right)^{n} n ! \\
& \tilde{\alpha}(x)=\frac{\alpha_{0}}{1-b_{0} \alpha_{0} x}, x=\ln \left(\frac{Q^{2}}{k^{2}}\right), y=\ln \left(\frac{k^{\prime 2}}{k^{2}}\right) .
\end{aligned}
$$

Here $\tilde{\alpha}(x)$ is simply the one loop running coupling for the momentum $k$. The trivial expansion in the series over $x$ now gives

$$
F_{n}=\int_{0}^{\infty} \frac{e^{-y}}{\left(1+b_{0} \alpha_{0} y\right)^{n+1}} d y
$$

Of course this $F_{n}\left(\alpha_{0}\right)$ may be expanded in the asymptotic series in $\alpha_{0}$. For very large number $n$ the integral in (43) is even further simplified. For example for the last $F_{n}$-s which one is allowed to work with (with $n \approx N_{I R}$ (37)) the expression (43) reduces to almost trivial result $F_{N_{I R}}=1 / 3$.

\section{Event shape renormalons and conclusions}

The IR renormalons for the event shape variables have became the popular topic of high energy physics in last couple of years (see e.g [20] and references therein). It was recognized that among the experimental values, which can not be directly related to the usual operator product expansion in Euclidean domain, one may find the quantities for which the soft gluon contribution is much less suppressed than in (1). In particular, the corresponding integral may even contain only the first power of $k$ instead of the usual fourth power (11)

$$
R_{E S}=\int_{k \ll Q} \alpha(k) \frac{d k}{Q}
$$

It is clear, that such contribution will generate the very large $\sim \Lambda / Q$ nonperturbative correction. Like for other renormalons this correction currently can not be found within $a b$ initio analytic calculation. One may suppose however, (as we have done in fact in (37)) that the smallest term of the series associated with (44) may be considered as the proper order of magnitude estimate of this $\sim \Lambda / Q$ correction. Then in order to be able to extract from the experiment the pure nonperturbative part one should calculate explicitly the

$$
N_{E S}(Q)=\frac{1}{2 b_{0} \alpha(Q)}
$$

terms of perturbation theory. Although, formally this number $N_{E S}(Q)$ still is parametrically very large $\sim 1 / \alpha$, it is 4 times smaller than the corresponding value for the usual IR renormalon (37). 
Moreover, it is usually assumed [17] that the actual $\sim \Lambda / Q$ corrections may be sufficiently enhanced compared to the smallest term of the series. Suppose that this is just the case and the nonperturbative $\Lambda / Q$ contribution is in $C$ times enhanced compared to the smallest term with some $C \gg 1$. In this case one may easily modify the eq. (45)

$$
N_{E S}(Q)=\frac{1}{2 b_{0} \alpha(Q)}-\sqrt{\frac{\ln (C)}{b_{0} \alpha(Q)}} .
$$

While writing this formula we still have assumed that $b_{0} \alpha \ln (C) \ll 1$. Naturally the difference $\Delta N_{E S}$ between (46) and (45) became relatively less important for smaller coupling $\Delta N_{E S} / N_{E S} \sim \sqrt{\alpha \ln (C)}$.

Now let us return to the main subject of our paper and consider the high order corrections to the Event-Shape renormalon. To obtain the Event-Shape renormalon (44) one has to consider some rather special characteristic of the jet distribution (thrust for example in [17]). Therefore, it is natural to suppose that the high order corrections to the effective coupling in (44) are still gathered by the traditional renormalons, and especially by the UV one. Under this assumption, repeating again the calculation (22,28) one gets for the $N$-th term of the expansion of (44)

$$
\left\{R_{E S}\right\}_{N} \sim\left(2 b_{0} \alpha\right)^{N} N^{\lambda} N !\left\{\sum_{m=1} r_{m}\right\},
$$

where the multiloop corrections $r_{m}$ are expected to behave like

$$
r_{m} \sim \frac{\beta_{m}}{\left(2 b_{0}\right)^{m} m !} \sim(-1 / 2)^{m}
$$

Thus we see that the series of multiloop corrections to the Event-Shape renormalon will naturally be described by the simple summable geometrical progression. However, this stability of the Event-Shape renormalon also reflects the fact that the best accuracy which in principle may be achieved within the pure perturbative calculation for (44) is much worse than for the usual IR renormalon $\left(\sim \Lambda / Q\right.$ instead of $\sim(\Lambda / Q)^{4}$ respectively ).

To conclude, the goal of this paper was mainly to outline the problems which should be solved upon taking into account the multiloop corrections to the renormalon chain. In particular, we have shown that these high order corrections play very different role for UV, IR and Event-Shape renormalons. The renormalon contribution to high order terms of perturbation theory for Event Shape variables are most hugely divergent compared to other renormalons. However, just the Event-Shapes renormalons turns out to be most stable under taking into account the multiloop corrections to renormalon chain. On the opposite side, the traditional IR renormalon is much weaker than other renormalons, but, as we have shown in Section 5, it is most sensitive to the inclusion of complicated insertions into the chain (divergent geometrical progression). Nevertheless, one is still able to associate with the IR renormalon some perturbation-theory-like series. Only the coefficients of this series should themselves be the sums of Borel summable series in $\alpha_{s}$. 
The special importance of the traditional IR renormalon in QCD is due to the fact that it gives the principal limitation on the accuracy of pure perturbative calculations. Finally, the resummation of high order corrections to any type of renormalons is sufficiently based on the use of UV renormalon. In Section 3 we have shown, how taking into account of the high order corrections to the single UV renormalon chain may be reduced to solution of a simple self-consistent equation. A considerable progress have been achieved last years in calculating the UV renormalon asymptotics with many hard photon(gluon) chains [12, 22, 30]. Nevertheless, in Section 4 we speculate about the possibility to change the asymptotics again by taking into account the multi-loop insertions into this many chain renormalon.

The most direct use of renormalons, as well as any other asymptotic estimates, will be to predict the value of $n+1$-st term of perturbation theory after the explicit calculation of $n$ terms. However, contrary to the case of Instantons [26], the overall amplitude of renormalon itself can not be found without accurate multiloop calculation. Moreover, even if the asymptotics of perturbation theory will be found, the corrections to it may be also very important. For example in QCD 29] the corrections to the asymptotics are of the order of $\sim N_{c}^{2} / N$ which makes practically useless the asymptotic estimates in this case. Another application of renormalons may be to use some kind of renormalon resummation in order to extract the pure nonperturbative-QCD contributions from the real high-energy experiment. However, in this case one may hope either on the possible (occasional) huge enhancement of the nonperturbative corrections, or to wait a few decades before the needed number of terms of perturbation theory (37,45,46) will be calculated explicitly.

This work was supported by the Russian Foundation for Fundamental Research under Grant 95-02-04607a. The work of S.F. has been supported by the INTAS Grant 932492-ext within the program of ICFPM of support for young scientists. P.S. thanks the hospitality of the Instituut Lorentz, Leiden, where this paper was completed.

\section{References}

[1] G.'t Hooft, in: The why's of subnuclear physics, (Erice, 1977), ed. A. Zichichi, (Plenum New York 1977).

[2] G.Parisi, Nucl. Phys. B150 (1979) 163.

[3] F. David, Nucl. Phys. B234 (1984) 237.

[4] A.H. Mueller, Nucl. Phys. B250 (1985) 327.

[5] G. B. West, Phys. Rev. Lett. 67 (1991) 1388.

[6] L. S. Brown and L. G. Yaffe, Phys. Rev. D45 (1992) R398.

L. S. Brown, L. G. Yaffe, and C. Zhai, Phys. Rev. D46 (1992) 4712. 
[7] V.I. Zakharov, Nucl. Phys., B385 (1992) 452.

[8] G. Grunberg, Phys. Lett. B304 (1993) 183.

[9] A.H. Mueller, Phys. Lett. B308 (1993) 355.

[10] M. Beneke, Phys. Lett. B307 (1993) 154.

[11] D.J. Broadhurst, Z. Phys C58 (1993) 339.

[12] A.I. Vainshtein and V.I. Zakharov, Phys. Rev. Lett. 73 (1994) 1207; Phys. Rev. D54 (1996) 4039.

[13] C.N. Lovett-Turner and C.J. Maxwell, Nucl. Phys. B432 (1994) 147.

[14] M. Beneke and V.M. Braun, Phys. Lett. B348 (1995) 513.

P. Ball, M. Beneke and V.M. Braun, Nucl. Phys. B452 (1995) 563.

P. Ball, M. Beneke and V.M. Braun, Phys. Rev. D52 (1995) 3929.

[15] B. R. Webber, Phys. Lett. B399 (1994) 148.

[16] G. Korchemsky and G. Sterman, Nucl. Phys. B437 (1995) 415.

[17] R. Akhoury and V. I. Zakharov, Phys. Lett. B357 (1995) 646; Nucl. Phys. B465 (1996) 295.

[18] Yu. L. Dokshitzer, G. Marchesini, and B. R. Weber, Nucl. Phys. B469 (1996) 93.

[19] M. Beneke, V. M. Braun, L. Magnea hep-ph/9609266

[20] R. Akhoury and V. I. Zakharov hep-ph/9610492

[21] A.H. Mueller, private communication.

[22] M. Beneke, V.A. Smirnov, Nucl. Phys. B472 (1996) 529.

[23] S.V.Faleev and P.G.Silvestrov, preprint BudkerINP-95-86, hep-ph/9510343, to appear in the Proceedings of Quarks96, May 1996, Yaroslavl,Russia.

[24] N. J. Watson hep-ph/9606381

[25] M. Beneke, Nucl. Phys. B405 (1993) 424.

[26] L. N. Lipatov, Zh. Eksp. Teor. Fiz. 72 (1977) 411.

[27] I. I. Balitsky, Phys. Lett. B273 (1991) 282.

[28] S. V. Faleev, P. G. Silvestrov, Phys. Lett. A197 (1995) 372.

[29] S. V. Faleev, P. G. Silvestrov, Nucl. Phys. B463 (1996) 489.

[30] M. Beneke, V.M. Braun, N. Kivel hep-ph/9703389 